\title{
Candida isolation from peritoneal fluid: Its role in the outcome of patients with perforation peritonitis
}

\author{
Shyam Lal ${ }^{*}$, Vinod Kumar Singh, Suhas Agarwal \\ Department of General Surgery, ESIC-PGIMSR and Model Hospital, Basaidarapur, News Delhi, India
}

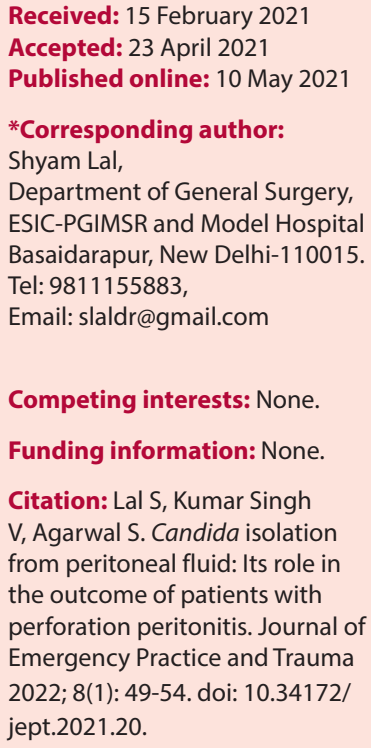

\begin{abstract}
Objective: Perforation peritonitis is a common surgical emergency which is treated by surgery and antibiotics. Candida isolation in peritoneal fluid and antifungal treatment is not a norm. The aim of this study was to determine the incidence of Candida in peritoneal fluid and its role in the outcome of patients with perforation peritonitis.

Methods: This prospective observational study was conducted on 70 patients with perforation peritonitis from October 2016 to February 2018. Intraoperatively, peritoneal fluid was taken and sent for microbiological culture and sensitivity. Perforation was managed according to the site of perforation and condition of bowel.

Results: The mean age of the patients was 38.74 years with male predominance $(58$, $82.85 \%)$. Forty-seven (67.14\%) patients had positive peritoneal cultures. Escherichia coli was the most common bacteria $(n=29)$, while Candida was found to be the most common fungi and was found in 18 patients. The incidence of Candida was higher in upper gastroduodenal perforation (30,42.85\%). Patients found positive for Candida had APACHE II severity score 10 or more which was higher than the rest of the patients. The mortality was higher in patients with positive peritoneal cultures (10/47) as compare to negative ones $(2 / 23, P<0.001)$. The mortality in mixed bacterial and fungal-positive cultures $(7 / 18)$ was also higher as compared to isolated bacterial culture $(3 / 29, P<0.001)$. The overall mortality rate was $17.14 \%$.

Conclusion: Patients with Candida positive peritoneal culture had a significant mortality and morbidity as compared to Candida negative. Peritoneal fluid culture and sensitivity for bacterial and fungal were helpful in the early diagnosis and treatment.

Keywords: Perforation peritonitis, Candida, Fungal peritonitis, Outcome, Primary peritonitis, Abdominal cavity, Laparotomy
\end{abstract}

\section{Introduction}

Perforation of the hollow viscus is the most common cause of peritonitis with grave consequences and it is attributed to microbial infection of peritoneal cavity. The mortality rate in perforation peritonitis ranges from $17 \%$ to $63 \%$ (1). The contaminating micro-organisms are poly-microbial which include gram negative bacilli and anaerobic bacteria. Recently, more emphasis has been on fungal co-infection (Candida peritonitis) as it is associated with worse outcomes $(2,3)$. The incidence of fungal peritonitis varies from $3 \%$ to $12 \%$ with high mortality (1). Epidemiological data over the last decades have shown that Candida peritonitis is frequent and has life threatening complication in critically ill patients $(4,5)$. Neither the Infectious Diseases Society of America (IDSA) guidelines (6) nor the European consensus (7) has expressed any clarification on Candida peritonitis management. Hence, peritoneal fluid culture for fungus is not a norm. However, some studies suggest that peritoneal fluid culture for bacterial and fungal in perforation peritonitis showed good outcome due to the early and appropriate treatment. Therefore, we performed this study to determine the incidence of Candida in peritoneal fluid and its role in the outcome of patients with perforation peritonitis.

\section{Methods}

This prospective observational study was conducted on 70 patients with perforation peritonitis in the Department of Surgery at ESIC- Post Graduate Institute of Medical Sciences and Research, New Delhi, India, from October 2016 to February 2018. The study protocol was approved by the institutional ethical committee (code-1377). Written inform consent was obtained from all the patients prior to surgery and enrolment. We included patients with the age range of 18 to 70 years who underwent exploratory laparotomy for spontaneous gastro-intestinal perforation. We exclude the patients of primary peritonitis, tertiary peritonitis, gynecological, trauma, and patients on antifungal treatment before the surgery.

Demographic data along with co-morbid conditions, 
preoperative clinical parameters, duration of fever, duration of abdominal pain, use of antibiotics and perforation-to-operation time interval were recorded. The diagnosis was made by clinical and radiological examination. Clinical conditions of patients were assessed by American Society of Anesthesiologists (ASA) grading, and Acute Physiology and Chronic Health Evaluation-II score (APACHE -II) score. Intraoperatively, the peritoneal fluid was taken and sent for gram stain, $\mathrm{KOH}$ (potassium hydroxide) mount and culture for aerobic bacteria and fungi. Blood and MacConkey agar were used for bacteria and Sabouraud dextrose agar was used for fungus. Fungi and bacteria were identified by standard laboratory methods. Anaerobic bacteria culture and species identification of fungus were not done. The intraoperative findings like size of the perforation, the amount and the nature of the contamination, as well as the intraoperative hemodynamic stability were recorded. Operative procedures were selected according to the location of perforation and intraoperative bowel condition. The initial choice of antibiotics was according to the institute protocol and relevant changes of antibiotics were carried out based on the culture and sensitivity. Candida positive (CP) peritoneal sample was defined as direst examination and/or culture growing Candida.

Issues related to mechanical ventilation, inotropic support, ICU stay and length of hospitalization were recorded. The postoperative complications like surgical site infections, wound dehiscence, intra-abdominal collection, chest complications, treatment, and death were recorded.

Data were entered into Microsoft excel sheet and statistical analyses were performed using the Statistical Package for the Social Sciences (SPSS, Version 12.0) for Windows. Comparisons of clinical characteristics and prognosis were made in patients with $\mathrm{CP}$ culture and patients with Candida-negative $(\mathrm{CN})$ culture. Continuous variables were analyzed by using mean and standard deviation (SD). Categorical variables were analyzed with proportions. Independent student's $t$ test was used to assess the significance of differences between groups of continuous variables and Fisher's exact test and Pearson's chi-square test were used for the categorical variables. A $P$ value of $\leq 0.05$ was considered statistically significant.

\section{Results}

This study was conducted on 70 patients with gastrointestinal perforation. Among the patients, 58 were males and 12 were females (M: F; 5:1). Out of 58 males, 16 were positive and 42 were negative, whereas out of 12 females, 2 were positive and 10 were negative $(P=0.718)$. Male patients outnumbered in both $\mathrm{CP}$ culture and) culture as compared to females (Table 1).

There was no significant difference in the age, gender, body mass index (BMI), American Society of Anesthesiologists (ASA) and co-morbidities among CP and $\mathrm{CN}$ patients. Patients having perforation to operation time of less than 48 hours in $\mathrm{CP}$ and $\mathrm{CN}$ were $6(33.33 \%)$ and $25(48.07 \%)$, respectively $(P<0.006)$, whereas $12(66.67 \%) \mathrm{CP}$ and $27(51.92 \%) \mathrm{CN}$ patients had a perforation to operation time of more than 48 hours. The findings were statistically significant $(P<0.016)$ (Table 2$)$.

$\mathrm{CP}$ patients had longer ICU stay. The mean ICU stay in $\mathrm{CP}$ and $\mathrm{CN}$ patients was $6.28 \pm 5.8$ and $1.37 \pm 2.35$ days, respectively, which was statistically significant $(P<0.0019)$. Additionally, the mean hospital stay was significantly higher in $\mathrm{CP}$ patients as compared to $\mathrm{CN}$ patients $(24.6 \pm 24.62$ versus $10.6 \pm 4.76$ days $(P<0.0002)$. Moreover, the CP patients had a higher mean score for APACHE II as compare to $\mathrm{CN}$ patients $(11.00 \pm 3.956$ versus $8.94 \pm 2.76 ; P<0.0409)$. This suggests that the Candida positivity was more in critically ill patients (Table 2).

In the study population, the site of perforation was found to be gastric in $14(20 \%)$ patients, duodenal in 16 $(22.8 \%)$ patients, jejunal in $6(8.57 \%)$ patients, Ileal in 19 (27.14\%) patients, appendicular in $11(15.71 \%)$ patients and caecal in $4(5.71 \%)$ patients, thereby indicating perforation to be more common in upper gastrointestinal tract (Table 1).

The micro-organisms isolated in peritoneal fluid culture and their antimicrobial susceptibility is shown in Tables 3 and 4. The commonest bacteria was Escherichia coli, isolated in 29 patients $(n=29)$ whereas the commonest fungi found was Candida in 18 (25.71\%) patients. Candida was found mostly in association with some or other bacteria i.e., in 17 out of 18 patients; however, it was isolated without any bacterial association in only a single patient of the 18 patients found positive for Candida.

Table 1. Site of perforation in relation to fungal positivity

\begin{tabular}{|c|c|c|c|c|c|c|c|c|}
\hline \multirow{2}{*}{ Site } & \multicolumn{3}{|c|}{ Candida positive } & \multicolumn{3}{|c|}{ Candida negative } & \multirow{2}{*}{ Total } & \multirow{2}{*}{$P$ value } \\
\hline & Male & Female & Total & Male & Female & Total & & \\
\hline Gastric & 3 & 1 & 4 & 7 & 3 & 10 & $14(20 \%)$ & \\
\hline Duodenal & 4 & 0 & 4 & 10 & 2 & 12 & $16(22.85)$ & \\
\hline Jejunum & 2 & 0 & 2 & 4 & 0 & 4 & $6(8.57 \%)$ & \\
\hline Ileal & 4 & 1 & 5 & 12 & 2 & 14 & $19(27.14 \%)$ & 0.988 \\
\hline Appendicular & 2 & 0 & 2 & 6 & 3 & 9 & $11(15.71 \%)$ & \\
\hline Caecal & 1 & 0 & 1 & 3 & 0 & 3 & $4(5.71 \%)$ & \\
\hline Total & 16 & 2 & 18 & 42 & 10 & 52 & $70(100)$ & \\
\hline
\end{tabular}


Table 2. Patient demographics and postoperative complications

\begin{tabular}{|c|c|c|c|}
\hline Variables & Candida Positive (CP) [n=18] & Candida Negative $(\mathrm{CN})[\mathrm{n}=52(\%)]$ & $P$ value \\
\hline Age (years, mean $\pm \mathrm{SD}$ ) & $36.608 \pm 13.936$ & $44.47 \pm 18.984$ & 0.0623 \\
\hline $\mathrm{BMI}($ mean $\pm \mathrm{SD})$ & $26.6 \pm 5.2$ & $25.5 \pm 6.7$ & 0.5291 \\
\hline \multicolumn{4}{|l|}{ Comorbidity } \\
\hline Diabetes mellitus & $6(33.33)$ & $14(26.92)$ & \multirow{4}{*}{0.7457} \\
\hline Hypertension & $6(33.33)$ & $12(23.08)$ & \\
\hline Respiratory disease & $3(16.67)$ & $4(7.69)$ & \\
\hline Tuberculosis & $7(38.89)$ & $8(15.38)$ & \\
\hline ASA grade $(4 \mathrm{E} / 5 \mathrm{E})$ & $18(100 \%)$ & $52(100 \%)$ & \\
\hline APACHE II $($ mean \pm SD $)$ & $11.00 \pm 3.956$ & $8.94 \pm 2.76$ & 0.0409 \\
\hline Perforation to operation time $<48 \mathrm{~h}$ & $6(33.33)$ & $25(48.07)$ & 0.0006 \\
\hline Perforation to operation time $>48 \mathrm{~h}$ & $12(66.67)$ & $27(51.92)$ & 0.016 \\
\hline ICU (days, mean \pm SD) & $6.28 \pm 5.8$ & $1.37 \pm 2.35$ & 0.0019 \\
\hline Hospital stay (days, mean \pm SD) & $24.6 \pm 24.622$ & $10.6 \pm 4.76$ & 0.0002 \\
\hline Mortality & $7(38.9 \%)$ & $5(9.6 \%)$ & 0.001 \\
\hline
\end{tabular}

Figures in parentheses denote percentage.

Abbreviations: APACHE: Acute Physiology and Chronic Health Evaluation; SD: standard deviation; ASA: American Society of Anesthesiologists; ICU: intensive care unit.

Table 3. Micro-organisms isolated in peritoneal fluid culture

\begin{tabular}{lcc}
\hline Micro-organism & Number & Percentage \\
\hline Escherichia coli & 29 & 50.87 \\
Klebsiella & 10 & 17.54 \\
Salmonella & 7 & 12.28 \\
Enterobacter & 6 & 10.53 \\
Pseudomonas & 5 & 8.77 \\
\hline
\end{tabular}

Table 4. Antimicrobial susceptibility of micro-organism isolated in peritoneal fluid

\begin{tabular}{lc}
\hline Antimicrobial & Percent \\
\hline Imipenem & 96.20 \\
Ceftriaxone & 88.00 \\
Sulbactam and cefoperazone & 68.6 \\
Amikacin & 62.80 \\
Cefotaxime & 57.30 \\
Ciprofloxacin & 45.20 \\
\hline
\end{tabular}

Morbidity in terms of superficial surgical site infections, intraabdominal abscesses, wound dehiscence and chest complications were higher in CP patients $(12 / 18 ; 66.7 \%)$ than in $\mathrm{CN}$ patients $(7 / 52 ; 13.5 \%)$ ( $\mathrm{CN}$ included bacteria positive and sterile samples). These findings were statistically significant $(P<0.001)$ (Figure 1$)$.

On microbiological evaluation of the samples of 70 patients, Candida (with or without bacterial association) grew in 18 cases (CP) only, bacteria grew in 29 patients and 23 samples came out to be sterile. Thus, 52 samples were negative for Candida. In total 10/47 (21.27\%) patients died, while $2 / 23(8.69 \%)$ patients died having sterile peritoneal fluid cultures. Three out of 29 (10.34\%) patients having positive cultures for bacteria only, died, whereas $7 / 18$ patients with positive cultures for mixed i.e., bacterial as well as fungal growths died $(38.90 \% ; P<0.001)$
The results were statistically significant (Table 5).

The mortality was higher in CP patients $(7 / 18,38.9 \%)$ as compare to $\mathrm{CN}$ patients $(5 / 52,9.60 \%)$. This finding was statistically significant $(P<0.001)$. The overall mortality rate was $17.14 \%$. Therefore, the outcome was significantly worse in patients with positive fungal culture as compared to patients with negative fungal culture.

\section{Discussion}

In a tropical country like India, perforation peritonitis is a common surgical emergency. It commonly affects young men in comparison to the studies from the west where the mean age is between $45-60$ years (8). The mean age in the present study was $38.74 \pm 13.0$ years which corroborates with other studies from India (8-10). Worldwide, it is predominant in males, $(8,10)$ and similar trends were present in our study with male to female ratio of 5:1.

Candida spp. are predominant pathogens and are found in $70 \%-90 \%$ of fungal peritonitis $(4,11)$. They are associated with considerable morbidity and mortality in critically ill patients (12). It is a commensal of the digestive tract, which leaks into the peritoneal cavity

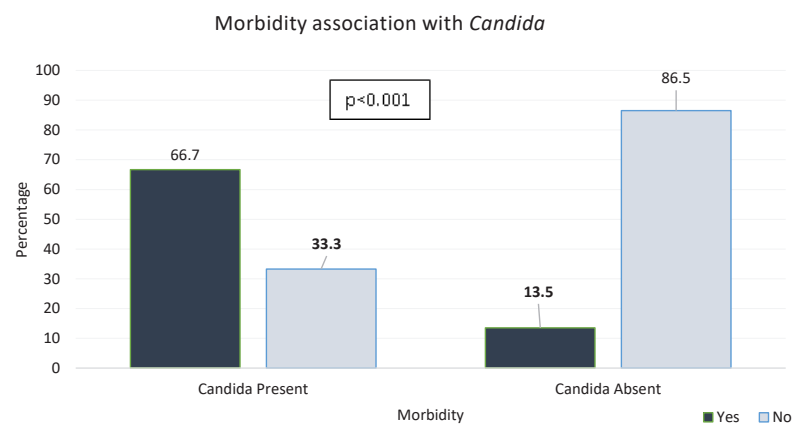

Figure 1. Morbidity associated with Candida peritonitis. 
Table 5. Distribution of patients according to bacterial and fungal culture and mortality

\begin{tabular}{|c|c|c|c|c|c|c|c|c|}
\hline & & \multicolumn{4}{|c|}{ Bacteria } & \multicolumn{2}{|c|}{ Mortality } & \multirow{2}{*}{$P$ value } \\
\hline & & No & Mortality & Yes & Mortality & No & Yes & \\
\hline \multirow{2}{*}{$\begin{array}{l}\text { Candida } \\
\text { infection }\end{array}$} & Present 18 & 1 & $0(0)$ & $17(94.4)$ & $7(41)$ & $11(61.1)$ & 7 (38.9) & \multirow{3}{*}{$<0.001$} \\
\hline & Absent 52 & 23 & $2(8.69)$ & $29(55.8)$ & $3(10)$ & 47 (90.4) & $5(9.6)$ & \\
\hline Total & 70 & 24 & $2(8.69)$ & $46(65.7)$ & $10(21.73)$ & $58(82.9)$ & $12(17.1)$ & \\
\hline
\end{tabular}

after perforation and results in intra-abdominal Candida infection $(5,13)$. The rate of Candida isolation from peritoneal fluid ranges from $1 \%-38 \%$ in gastrointestinal perforation $(2,8,10)$. The incidence in our study was $25.71 \%$. The causes for variations in the rate of Candida isolation could be related to different patient populations and methods used or a combination of both.

Candida peritonitis was more common in patients presenting with perforation to operation time of more than 48 hours thereby showing association with significantly higher mortality $(3,8)$. Patients having perforation to operation time of less than 48 hours in $\mathrm{CP}$ and $\mathrm{CN}$ were $6(33.33 \%)$ and $25(48.07 \%)$, respectively $(P<0.006)$, whereas 12 (66.67\%) CP and 27 (51.92\%) CN patients had a perforation to operation time of more than 48 hours $(P<0.016)$. This might be due to the time required for the Candida to multiply and become detectable more often. Patients with Candida peritonitis had increased amount of contamination, shock, and required preoperative inotropic and mechanical support (8).

Candida peritonitis is more common in critically ill patients. APACHE II score was used to assess the severity of peritonitis. A high APACHE II score was reported as an independent risk factor for subsequent Candida peritonitis and mortality $(2,3,14,15)$. In our study, the mean of APACHE II score was higher in CP patients as compared to $\mathrm{CN}$ patients $(11.00 \pm 3.956$ vs $8.94 \pm 2.76 ; P<0.0409)$. Therefore, patients who required ICU admissions, longer hospital stay, and significant post-operative morbidity and mortality were found to have Candida peritonitis $(3,8,10)$. The Candida peritonitis is more common in upper gastrointestinal perforation as reported in various studies such as Shan (2) 43.4\%, Jindal (10) $70.5 \%$, and Katlana (16) $45.2 \%$. In our study, $42.86 \%$ of patients had upper gastrointestinal perforation.

Morbidity in terms of superficial surgical site infections, intra-abdominal abscesses, wound dehiscence and chest complications were higher in CP patients $(12 / 18,66.7 \%)$ as compared to $\mathrm{CN}$ patients $(7 / 52,13.5 \% ; P<0.001)$. Similar result was reported in previous studies $(2,3,8,10)$. These studies indicate poor outcome associated with Candida peritonitis.

A significant higher mortality was reported in different studies concerning patients with Candida peritonitis $(8,9)$. Our findings were also in line with other studies which state that higher mortality rates were observed more in: (a) patients with positive culture in comparison to those with sterile culture, $(b)$ mixed positive (Candida along with bacterial) culture as compared to bacterial positive culture only, and (c) patients positive for Candida as compared to patients negative for Candida. Therefore, the Candida co-infection appears to be a reason leading to poor prognosis in such patients. We found that $\mathrm{CP}$ in peritoneal fluid culture was associated with a worse outcome in patients with perforation peritonitis. The mortality reported by Dupont et al, Pramod et al and Prakash et al was 43\%,50\%,77\%, respectively in Candida positive peritoneal fluid $(4,8,9)$. In our study, the mortality was higher in CP patients $7 / 18$ (38.9\%) as compared to CN patients $5 / 52(9.60 \% ; P<0.001)$. The overall mortality rate in the present study was $17.14 \%$. High mortality was observed in patients positive for Candida as these patients presented late, i.e. having more than 48 hours of perforation to operation time, with higher APACHE II score, septic shock, and required more ICU admission.

Prognosis of a patient with perforation of an abdominal viscera is generally poor. Management of these patients requires surgery along with the use of broad-spectrum antibiotics but the role of antifungal treatment remains controversial and practice guidelines are conflicting $(6,7)$. Some studies have demonstrated the advantage of early administration (within 48 hours) of antifungal treatment along with management of bacteria and primary foci of infection (1-3,8-11,17-19), whereas others studies, including a randomized control trial, have not shown the survival benefit of it even in critically ill patients (20-23). In this regard, there is not a final conclusion for this challenging and controversial issue $(10,12,24)$. Furthermore, the mortality for Candida mono-infection and Candida-bacterial co-infections was comparable in various studies, indicating that Candida spp. are significant pathogens within the abdominal cavity rather than innocent bystanders. However, in spite of treatment controversy, antifungal drugs are commonly prescribed in severely ill patients. These include use of prolong antibiotics, small bowel surgery, anastomotic leaks, the need for reoperation, pancreatitis, multi-organ failure, poor clinical and physical condition, mechanically ventilated patients and parenteral nutrition (21).

The results of the present study and other researches $(9,17,24)$ raise the possibility that the occurrence of intraabdominal Candida is associated with higher morbidity and mortality. Peritoneal fluid culture in perforation peritonitis for bacteria and fungi are easily available. Therefore, till management recommendations from studies on larger populations come out, it would be nice to recommend on the basis of our results that peritoneal fluid culture for bacterial and fungi should be done in 
all patients undergoing laparotomy in order to facilitate the diagnosis and treatment of the causative organism at the earliest time possible. High mortality observed in fungal co-infection patients can be minimized by early recognition and treatment.

\section{Conclusion}

Candida peritonitis was common in patients with delayed presentation, septic shock, and higher APACHE -II score. Candida peritonitis can lead to longer hospital stay, more surgical site infections, and significantly higher morbidity and mortality rate in comparison to patients with negative fungal culture. Fungal and bacterial cultures have to be considered in critically ill patients for adequate and timely treatment.

\section{Authors' Contribution}

Concept and design of the study, acquisition and interpretation of data, along with drafting the article or revising it critically for important intellectual content was done by SL, VKS and SA. All authors approved final version of manuscript.

\section{Ethical issues}

This study was approved by the ethics committee (code-1377) of ESIC- Post Graduate Institute of Medical Sciences and Research, New Delhi, India, which is affiliated to Guru Gobind Singh Indraprastha University, New Delhi.

\section{References}

1. Carneiro HA, Mavrakis A, Mylonakis E. Candida peritonitis: an update on the latest research and treatments. World J Surg 2011; 35(12): 2650-9. doi: 10.1007/s00268011-1305-2.

2. Shan YS, Hsu HP, Hsieh YH, Sy ED, Lee JC, Lin PW. Significance of intraoperative peritoneal culture of fungus in perforated peptic ulcer. Br J Surg 2003; 90(10): 1215-9. doi: 10.1002/bjs.4267.

3. Lee SC, Fung CP, Chen HY, Li CT, Jwo SC, Hung YB, et al. Candida peritonitis due to peptic ulcer perforation: incidence rate, risk factors, prognosis and susceptibility to fluconazole and amphotericin B. Diagn Microbiol Infect Dis 2002; 44(1): 23-7. doi: 10.1016/s0732-8893(02)00419-4.

4. Dupont H, Bourichon A, Paugam-Burtz C, Mantz J, Desmonts JM. Can yeast isolation in peritoneal fluid be predicted in intensive care unit patients with peritonitis? Crit Care Med 2003; 31(3): 752-7. doi: 10.1097/01. ccm.0000053525.49267.77.

5. Montravers P, Dupont H, Eggimann P. Intra-abdominal candidiasis: the guidelines-forgotten non-candidemic invasive candidiasis. Intensive Care Med 2013; 39(12): 2226-30. doi: 10.1007/s00134-013-3134-2.

6. Pappas PG, Kauffman CA, Andes DR, Clancy CJ, Marr KA, Ostrosky-Zeichner L, et al. Clinical practice guideline for the management of candidiasis: 2016 update by the Infectious Diseases Society of America. Clin Infect Dis 2016; 62(4): e1-50. doi: 10.1093/cid/civ933.

7. Cornely OA, Bassetti M, Calandra T, Garbino J, Kullberg $\mathrm{BJ}$, Lortholary $\mathrm{O}$, et al. ESCMID ${ }^{*}$ guideline for the diagnosis and management of Candida diseases 2012: nonneutropenic adult patients. Clin Microbiol Infect 2012; 18 Suppl 7: 19-37. doi: 10.1111/1469-0691.12039.

8. Pramod J, Vijayakumar C, Srinivasan K, Maroju NK, Raj
Kumar N, Balasubramaniyan G. Clinical significance of Candida in an intraoperative peritoneal specimen with perforation peritonitis: an institutional perspective. Cureus 2018; 10(3): e2275. doi: 10.7759/cureus.2275.

9. Prakash A, Sharma D, Saxena A, Somashekar U, Khare N, Mishra A, et al. Effect of Candida infection on outcome in patients with perforation peritonitis. Indian J Gastroenterol 2008; 27(3): 107-9.

10. Jindal N, Arora S, Pathania S. Fungal culture positivity in patients with perforation peritonitis. J Clin Diagn Res 2015; 9(6): DC01-3. doi: 10.7860/jcdr/2015/13189.6050.

11. Zhan J, Shu G, Yuan L, Zhu J, Xie B. Clinical features of fungal peritonitis with Candida albicans infection after gastric and duodenal perforation. Emerg Med (Los Angel) 2015; 5(4): 264. doi: 10.4172/2165-7548.1000264.

12. Knitsch W, Vincent JL, Utzolino S, François B, Dinya T, Dimopoulos G, et al. A randomized, placebo-controlled trial of preemptive antifungal therapy for the prevention of invasive candidiasis following gastrointestinal surgery for intra-abdominal infections. Clin Infect Dis 2015; 61(11): 1671-8. doi: 10.1093/cid/civ707.

13. Johnson DW, Cobb JP. Candida infection and colonization in critically ill surgical patients. Virulence 2010; 1(5): 355-6. doi: 10.4161/viru.1.5.13254.

14. Hsu FC, Lin PC, Chi CY, Ho MW, Ho CM, Wang JH. Prognostic factors for patients with culture-positive Candida infection undergoing abdominal surgery. J Microbiol Immunol Infect 2009; 42(5): 378-84.

15. Dupont H, Guilbart M, Ntouba A, Perquin M, Petiot S, Regimbeau JM, et al. Can yeast isolation be predicted in complicated secondary non-postoperative intra-abdominal infections? Crit Care 2015; 19(1): 60. doi: 10.1186/s13054015-0790-3.

16. Katlana A, Vyas AK, Jain R, Rathi A, Sharma NK, Yadav $\mathrm{AK}$, et al. Incidence and significance of intra-operative peritoneal fluid fungal culture in patients of perforated peptic ulcers. J Med Sci Clin Res 2017; 5(3): 18987-91. doi: 10.18535/jmscr/v5i3.110.

17. Bassetti M, Merelli M, Ansaldi F, de Florentiis D, Sartor A, Scarparo C, et al. Clinical and therapeutic aspects of candidemia: a five year single centre study. PLoS One 2015; 10(5): e0127534. doi: 10.1371/journal.pone.0127534.

18. Bassetti M, Righi E, Montravers P, Cornely OA. What has changed in the treatment of invasive candidiasis? a look at the past 10 years and ahead. J Antimicrob Chemother 2018; 73(Suppl 1): i14-i25. doi: 10.1093/jac/dkx445.

19. Vergidis P, Clancy CJ, Shields RK, Park SY, Wildfeuer $\mathrm{BN}$, Simmons RL, et al. Intra-abdominal candidiasis: the importance of early source control and antifungal treatment. PLoS One 2016; 11(4): e0153247. doi: 10.1371/ journal.pone.0153247.

20. Grim SA, Berger K, Teng C, Gupta S, Layden JE, Janda WM, et al. Timing of susceptibility-based antifungal drug administration in patients with Candida bloodstream infection: correlation with outcomes. J Antimicrob Chemother 2012; 67(3): 707-14. doi: 10.1093/jac/dkr511.

21. Timsit JF, Azoulay E, Schwebel C, Charles PE, Cornet $\mathrm{M}$, Souweine B, et al. Empirical micafungin treatment and survival without invasive fungal infection in adults with ICU-acquired sepsis, Candida colonization, and multiple organ failure: the EMPIRICUS randomized clinical trial. JAMA 2016; 316(15): 1555-64. doi: 10.1001/ jama.2016.14655. 
22. Khoury W, Szold O, Soffer D, Kariv Y, Wasserlauf R, Klausner JM, et al. Prophylactic fluconazole does not improve outcome in patients with purulent and fecal peritonitis due to lower gastrointestinal perforation. Am Surg 2010; 76(2): 197-202.

23. Li WS, Lee CH, Liu JW. Antifungal therapy did not improve outcomes including 30-day all-cause mortality in patients suffering community-acquired perforated peptic ulcerassociated peritonitis with Candida species isolated from their peritoneal fluid. J Microbiol Immunol Infect 2017; 50(3): 370-6. doi: 10.1016/j.jmii.2015.07.004.

24. Montravers P, Perrigault PF, Timsit JF, Mira JP, Lortholary $\mathrm{O}$, Leroy $\mathrm{O}$, et al. Antifungal therapy for patients with proven or suspected Candida peritonitis: Amarcand2, a prospective cohort study in French intensive care units. Clin Microbiol Infect 2017; 23(2): 117.e1-117.e8. doi: 10.1016/j.cmi.2016.10.001. 